\title{
Local Fatigue Evaluation in PZT Thin Films with Nanoparticles by Piezoresponse Force Microscopy
}

\author{
B. S. Li \\ Material and Reliability, ASMPT, Singapore 768924 \\ Correspondence should be addressed to B.S. Li, bsli@asmpt.com \\ Received 28 June 2011; Accepted 22 August 2011 \\ Academic Editor: Tao Li
}

Copyright () 2012 B. S. Li. This is an open access article distributed under the Creative Commons Attribution License, which permits unrestricted use, distribution, and reproduction in any medium, provided the original work is properly cited.

Lead zirconate titanate (PZT) thin films with the morphotropic phase boundary composition $(\mathrm{Zr} / \mathrm{Ti}=52 / 48)$ have been prepared using a modified diol-based sol-gel route by introducing 1-5 mol\% barium titanate (BT) nanoseeds into the precursor solution on platinized silicon substrates $\left(\mathrm{Pt} / \mathrm{Ti} / \mathrm{SiO}_{2} / \mathrm{Si}\right)$. Macroscopic electric properties of PZT film with nanoparticle showed a significant improvement of ferroelectric properties. This work aims at the systematic study of the local switching polarization behavior during fatigue in PZT films with and without nanoparticles by using very recent developed scanning piezoelectric microscopy (SPM). We show that the local fatigue performance, which is characterized by variations of local piezoloop with electric cycles, is significantly improved by adding some nanoseeds. It has been verified by scanning electron microscope (SEM) that the film grain morphology changes from columnar to granular structure with the addition of the nanoseeds. On the other hand, the existence of $\mathrm{Pt}_{x} \mathrm{~Pb}$ transition phase, which existed in interface at early crystallization stage of pure PZT thin film, deteriorates the property of the interface. These microstructures and the interfaces of these films significantly affect the electrons injection occurred on the interfaces. The domain wall pinning induced by injected electrons and the succeeding penetration into the films is discussed to explain the fatigue performance.

\section{Introduction}

Lead zirconate titanate (PZT) thin film has been a good candidate for application in ferroelectric materials in nonvolatile ferroelectric random access memory (FERAM) due to its excellent ferroelectric properties $[1,2]$. One prerequisite property of PZT thin films for ferroelectric devices is the microscopic perfection of the polarization switching, defined as the symmetric switching between two opposite polarization states. The occurrence of polarization fatigue, which is the decrease of polarization during repeated electrical pulses, has been an obstacle for full commercialization of the PZT thin films, especially for applications of PZT thin films on Pt electrode coated substrates. As such, extensive studies have been carried out to disclose the microscopic fatigue nature in PZT thin films [3-10]. Oxygen vacancies, which are very easy to form during crystallization process and the most mobile point defects in perovskite material like PZT, are deemed to be a major factor in fatigue. Scott et al. argued that the transition of oxygen vacancies into two-dimensional planar arrays is a fatigue mechanism in perovskite ferroelectrics
$[3,5]$. Park and Chadi investigated atomic relaxations around oxygen-vacancy defects in ferroelectric perovskite $\mathrm{PbTiO}_{3}$ through first-principles pseudopotential total energy calculations [6]. The atomic relaxations around oxygen vacancies may result in a tail-to-tail polarization and thus favor the charge trapping and domain pinning. Lupascu and Rabe directly observed point defect clustering in perovskite ferroelectrics during cyclic electrical pulses [7]. It seems, from these data, that the redistribution of the point defect (mainly oxygen vacancies) plays a crucial role in fatigue mechanism. However, there are some arguments against this opinion which are summarized in [8]:

(1) Doping is always used to control the oxygen vacancy concentration, but the experimental results on the effect of doping of PZT films on fatigue are contradictory.

(2) The activation energy which corresponds to the temperature dependence of the fatigue rate $(0.05 \mathrm{eV})$ is much smaller than the typical one of the oxygen vacancy mobility in perovskites $(1 \mathrm{eV})$. 
Considering the above arguments, one may draw the conclusion that in fatigue mechanism, the crucial contributing factor was not the redistribution of the oxygen vacancies. Studies reveal that charge carriers could also influence fatigue. An apparent polarization attenuation exists in PZT and $\mathrm{BaTiO}_{3}$ when ultraviolet illumination is used to generate electron and hole pairs [9]. When a sample is fatigued, electrons and holes injected from the electrodes into the ferroelectric film can be trapped at deep levels and immobilized producing as a simple charged defect [8]. These defects associated with the bound charges of the domain walls pin the reorientation of the domains during fatigue. Cillessen et al. demonstrated an electron injection model occurred in near-electrode region [10]. According to this model, the coercive field of the film will increase if the near-electrode electron injection is present. Du and Chen observed improvement of fatigue performance in PZT films when using $\mathrm{P}$ type silicon as the top electrode, and they attributed the improved fatigue endurance to absence of the electron penetration [11]. These findings demonstrate that electrons injection during fatigue plays a very important role in the fatigue performance of PZT films.

In our previous work $[12,13]$, a novel approach to improve microstructure and decrease the processing temperature of PZT films on Pt/Si by sol-gel was developed. This approach introduced small amounts of perovskite nanoparticles into the precursor, and the films prepared by seeded precursor show not only improved microstructure and ferroelectric properties, but also fatigue endurance. Our results show that an alternative to improve the fatigue endurance by adding nanoseeds into the films has been found. However, systematic studies on the seeding effect on fatigue performance at a nanoscale level have not been conducted yet, and the mechanism of this improvement remains to be discovered.

It should be noted that the fatigue mechanisms mentioned only consider the variations in macroscopic hysteresis loops before and after fatigue. In fact, this characterization could not define the microscopic fatigue performance and possible imprint along polarization axis well. Recently, piezoforce microscopy (PFM) has been intensively used to study the domain variations in ferroelectrics due to its significant advantage for monitoring domain features and ability for investigating local piezoresponse with piezoloop [14]. Furthermore, compared with macroscopic hysteresis loops, the microscopic piezoloop may well define the imprint phenomenon along the polarization axis due to its displacementdepended nature. In contrast, this kind of imprint cannot be detected in macroscopic measurements of ferroelectric polarization, because an arbitrary integration constant is used in the polarization measurement [15]. Therefore, in this work, PFM is utilized to analyze the local fatigue performances in three kinds of PZT ferroelectric thin films. The electron injection [16] and the succeeded domain pinning by the penetrated electrons were investigated in microregion by piezoloops. The diverse domain pinning processes in these films during fatigue were reported here, and a possible explanation was suggested.

\section{Experimental Procedure}

The PZT precursor solution was prepared according to the stoichiometric formula $\mathrm{Pb}\left(\mathrm{Zr}_{0.52} \mathrm{Ti}_{0.48}\right) \mathrm{O}_{3}$. No excess lead was added. The starting reagents were lead acetate trihydrate, titanium diisopropoxide bisacetylacetonate, and zirconium acetylacetonate. A $0.4 \mathrm{M}$ stock solution was used for the PZT thin-film deposition. The viscosity of the solutions with and without seeds was adjusted to $0.012 \mathrm{~Pa}$.

Ultra fine barium titanate (BT) powders $(20 \sim 80 \mathrm{~nm})$ were used as seeds. BT seed powder was prepared by a direct hydrolysis synthesis in which titanium alkoxide mixed into barium hydroxide solution at $90^{\circ} \mathrm{C}$. Perovskite phase was directly formed after drying the powder at room temperature. The BT powder was milled and dispersed with a suitable solvent and dispersant (Dispex A40; Allied Colloids, Ltdammonium salt of a polycarboxylic acid) for $5 \mathrm{~h}$ using a vibrator mill. The suspension of BT seeds was mixed with the PZT precursor sol according to certain ratios. The weight ratios of BT seeds in PZT sol were $1 \%$ and 5\%, respectively. The sol was processed with vigorous stirring and followed by a 20 min ultrasonic treatment. The stability of the PZT suspension sols was measured with a rheometer.

PZT films were deposited on $\mathrm{Pt} / \mathrm{Ti} / \mathrm{SiO}_{2} / \mathrm{Si}$ multilayered substrates by dip-coating. The substrate was dipped and withdrawn from the precursor sol at a constant rate of $0.17 \mathrm{~cm} / \mathrm{s}$. The resulting wet films were dried on a hot plate at $300^{\circ} \mathrm{C}$ for $1 \mathrm{~min}$ to remove residual organics. After deposition of four layers, the films were fired at $550^{\circ} \mathrm{C}$ for $30 \mathrm{~min}$ in air. The PZT thin films with $1 \mathrm{wt} \%$ and $5 \mathrm{wt} \%$ BT seeds are briefly called PZT-BT1 and PZT-BT5, respectively. The thickness of these films were about $400 \mathrm{~nm}$. The stoichiometry and the interfaces quality of the films were studied by Rutherford back scattering (RBS) spectrometry using a $\mathrm{He}^{+}$ beam at $1.6 \mathrm{MeV}$.

Surface morphology and domain structure inspection of the films was performed via so-called piezoforce microscopy (PFM) that relies on a local reverse piezoelectric effect exhibited by the film's surface. A modified commercial atomic force microscope (Multimode, Nanoscope IIIA, Digital Instruments) was used in the experiment to record synchronously two different features: topography and piezoresponse. A conductive $\mathrm{Ti}$ (1st layer) and Pt (2nd layer) coated tip cantilever (NSC14/Ti-Pt, MikroMasch) system was used for the application of external voltages and for effective piezocoefficient $\left(d_{\text {eff }}\right)$ measurements. The first harmonics of the cantilever vibration was detected under an ac voltage $(3 \mathrm{~V}$, $50 \mathrm{kHz}$ ) was applied between the grounded tip and the bottom electrode. In the piezoresponse image, domains with opposite polarization exhibit different contrast. Dark regions (hereafter referred to as negative domains) correspond to domains with polarization oriented towards the substrate, and bright regions (positive domains) refer to domains with polarization point at the free surface of the film. The local piezoelectric hysteresis loops (piezoloops) before and after fatigue were measured on a fixed point inside an unambiguous domain. The tip was put on the relative flat area to avoid the interference caused by $d_{31}$ and $d_{15}$ vibration. The loop acquisition consisted of applying a dc voltage, $V_{\mathrm{dc}}$, for a short time 


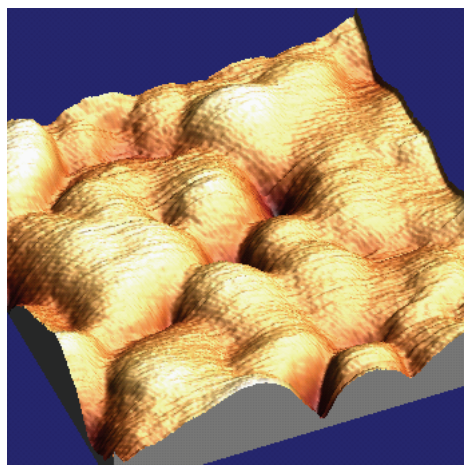

(a)

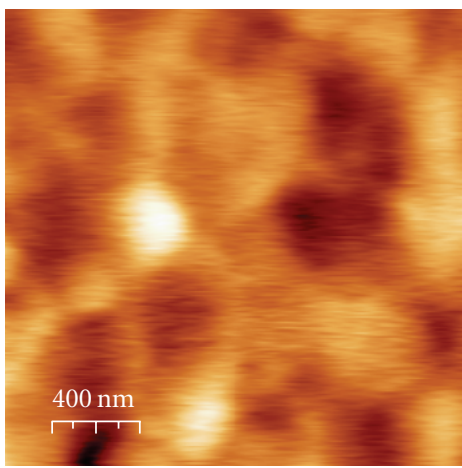

(d)

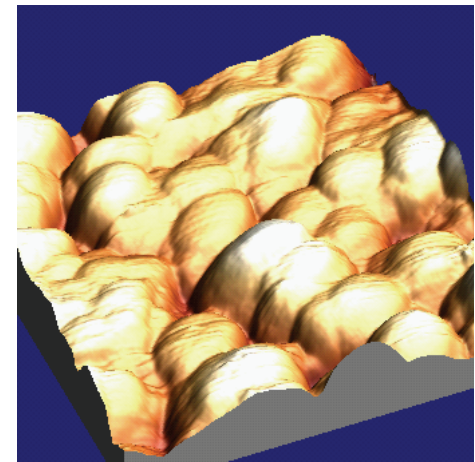

(b)

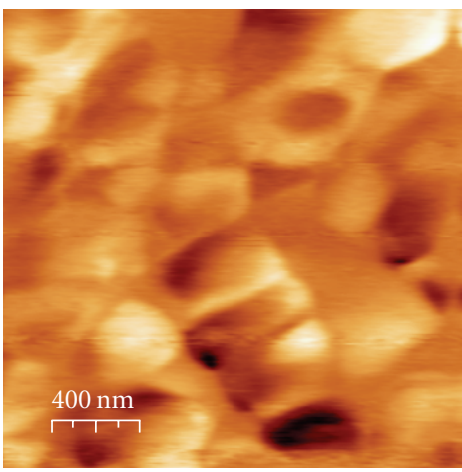

(e)

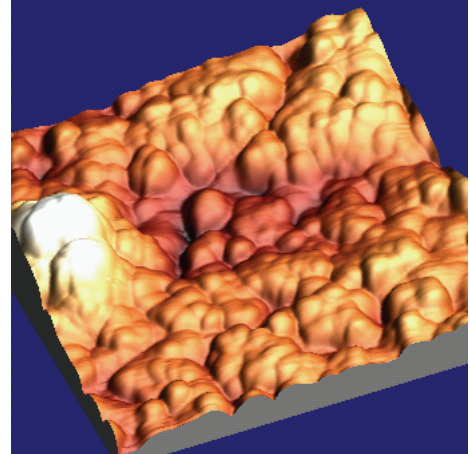

(c)

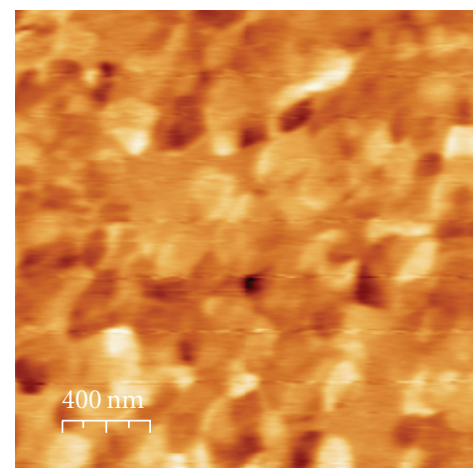

(f)

FIgure 1: Topographic images of PZT films without seeds (a), with 1\% BT seeds (b), with 5\% seeds (c), and corresponding piezoresponse images (d), (e), and (f) in three kinds of PZT thin films. The scale bar in topographic image is same as that in piezoresponse one.

$(\approx 1 \mathrm{~s})$ and measuring the actual remanent piezoelectricity when the $\mathrm{dc}$ voltage was reduced to zero for each piezoelectric measurement. The voltage was swept by $1 \mathrm{~V}$ increments in the range $-20 \mathrm{~V}<V_{\mathrm{dc}}<+20 \mathrm{~V}$. The frequency and amplitude of electric field for fatigue is $50 \mathrm{kHz}$ and 2 times of the corresponding coercive field, respectively.

\section{Results and Discussions}

Typical topography and piezoresponse images of three kinds of films are shown in Figure 1. Among these PZT films, pure PZT film shows the lowest surface roughness, while PZT-BT5 film presents the roughest one. The rms roughnesses are $2.01 \mathrm{~nm}, 4.93 \mathrm{~nm}$, and $7.33 \mathrm{~nm}$ for PZT, PZT-BT1, and PZTBT5 films, respectively. As suggested in [16], there were pyrochlore residue phase forming a uniform cover on PZT films. This may be the reason why the grains and the domain populations did not correlate each other.

It is also shown in Figure 1 that these films possessed different grain sizes. From the results, as addition of $1 \mathrm{wt} \%$ BT seeds did not result in a significant change in grain size, whereas a significant decreased in grain size was observed in PZT-BT5 film. Theoretically, nanoparticles added in the film can act as nucleation centers to favor crystallization process. Compared to the pure PZT films heated under the same conditions, the extent of crystallization of the perovskite structure was much higher [13]. The smaller grain in PZT-BT5 film is due to the presence of excessive nucleation center, which will increase the nucleation density and, therefore, to smaller grains.

3.1. Static Fatigue Studies. Figure 2 shows the piezoloops after different fatigue cycles in these films, which were measured consecutively for each film. The tip was immovable during the fatigue process, so it was briefly called "static fatigue". For pure PZT film, square-like piezoloop were observed when the measuring electric field was applied on the virgin film. There was an aggravation of the pinched shape to some degree with increasing of the fatigue switching cycles. After the $3 \times 10^{8}$ switching cycles, the ferroelectric behavior was entirely suppressed, and any expected correlation between the piezoresponse and the voltage applied disappeared, especially in the up polarized state. This illustrates that there were pinned domains in PZT film, because the domain size distribution during the growth process determined the hysteresis loop shape. Since a large ac field was applied in the fatigue experiment, the interior component of the PZT film was excited [17]. Thus, the pinned area was not just beneath the tip and localized in one domain. This might extend deep into the film due to the highly inhomogeneous and large electric field during fatigue experiment.

Note that PFM response has crystallographic dependence, which was dominated by $d_{33}$ value [18]. The relation between the coercive field and off-axis orientation of the grain could be approximately evaluated with [19]:

$$
E P \cos (\theta)=A,
$$




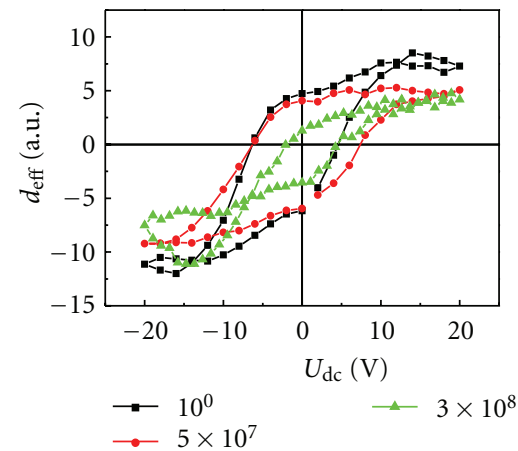

(a)

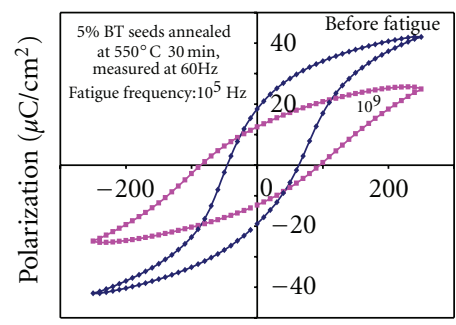

Applied field $(\mathrm{kV} / \mathrm{cm})$

(d)

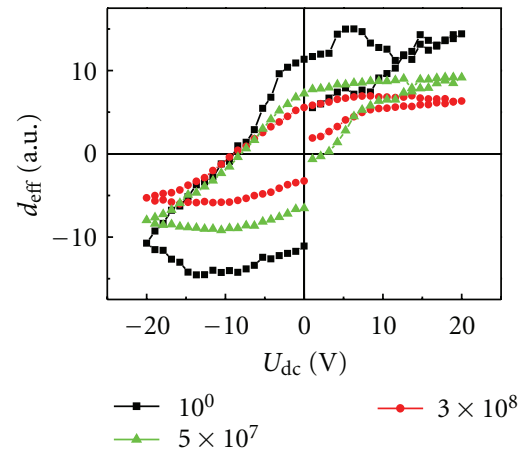

(b)

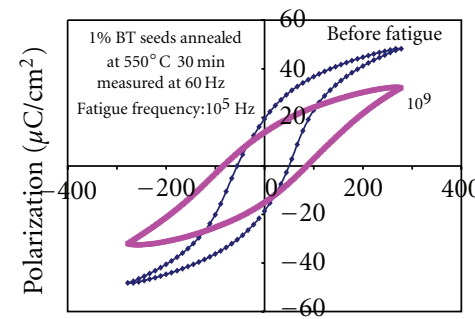

Applied field $(\mathrm{kV} / \mathrm{cm})$

(e)

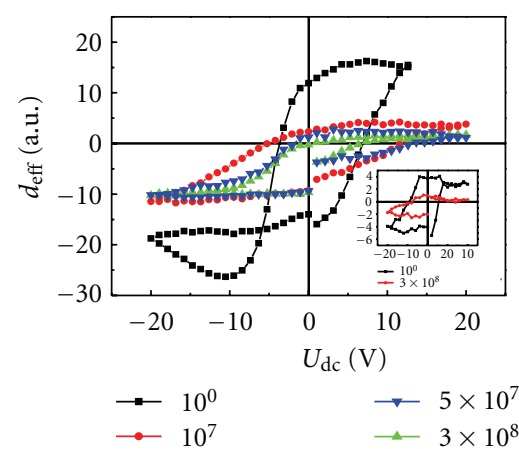

(c)

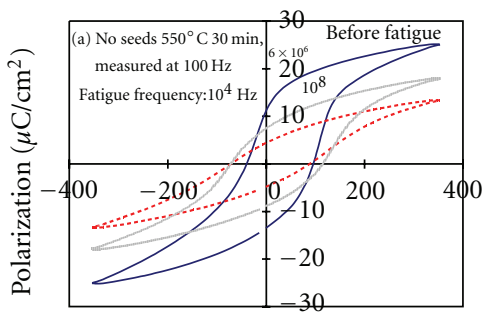

Applied field $(\mathrm{kV} / \mathrm{cm})$

(f)

Figure 2: Local (upper) and macroscopic (lower) piezoloops obtained in different PZT films of different fatigue cycles. (a) PZT-BT5 thin film, (b) PZT-BT1 film, and (c) PZT film. Inset in (c) shows variation of the piezoloops with electric cycles in PZT sample which is annealed in nitrogen at $600^{\circ} \mathrm{C}$ for 30 minutes.

where $E$ is electric field, $P$ is polarization, $\theta$ is off-axis angle and $A$ is a constant. Therefore, coercive field is expected to increase with the increase of off-axis angle, while polarization decreases to guarantee the product to be a constant. In present, it is obvious that increase of the coercive voltage (or decrease of the polarization) cannot be attributed to an offaxis orientation of the grain, since the product of coercive field and polarization significantly decreased after long-term fatigue cycles. Thus, this shows that the fatigue mechanism indeed involved a pinning process around domains. This is also an indication that under the probing location, the domain walls became clamped, inhibiting the growth of opposite domains so that a higher field was required to partly switch the domain into the opposite state. The slope of the piezoloop near the coercive field had a significant decrease, which indicates a difficult nucleation and expansion of the new domain.

Comparison of the loops in Figure 2(c) shows that the piezoloop presents apparent imprint after fatigue. It seemed that there was almost no positive displacement in PZT film after severe fatigue. During the measuring of piezoloop, it was believed that the switching begins with the nucleation of reverse domain seeds just underneath the tip. The newly growing domain then expanded to an equilibrium size reach to some depth in the film. The piezoelectric displacement can be expressed in the following way [20]:

$$
\begin{aligned}
d_{\text {eff }} & =\frac{1}{V_{\mathrm{ac}}} \int_{0}^{t} d_{33} E_{z} d z=\frac{d_{33}}{V_{\mathrm{ac}}}\left[\int_{0}^{l} E_{z} d z-\int_{l}^{t} E_{z} d z\right] \\
& =\frac{d_{33}}{V_{\mathrm{ac}}}[V(0)-2 V(l)],
\end{aligned}
$$

where $V(0)$ is the potential on the surface and $V(l)$ is the potential at the domain boundary. For the spherical model $V(l) \sim V(0) R /(R+l)[20]$, where $R$ is contact radius, $l$ is the distance from the center of the contact area. The surface potential $V(0)=V_{\text {tip }}=V_{\mathrm{dc}}+V_{\mathrm{ac}} \cos (\omega t)$. Then, the piezoelectric displacement is proportional to surface potential. Thus, the imprint of piezoloop can be explained by considering the large decrease of the $V_{\mathrm{dc}}$. It is highly probable that this decrease is caused by injection of electrons from the tip. The possible mechanism for electron injection is a thermoionic injection of negative charge carriers (electrons) through the tip, as described with [21]

$$
J=A T^{2} \exp \left(-\frac{\phi}{K T}\right)
$$

where $A$ and $K$ are the Richardson and Boltzmann constants, $T$ is the temperature, and $\phi$ is the effective barrier for electron injection. In other words, the increase in temperature along the fatigue process brings about an increase in current density, which will simultaneously decrease the effective barrier 


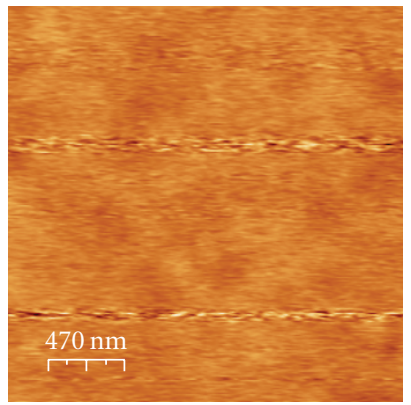

(a)

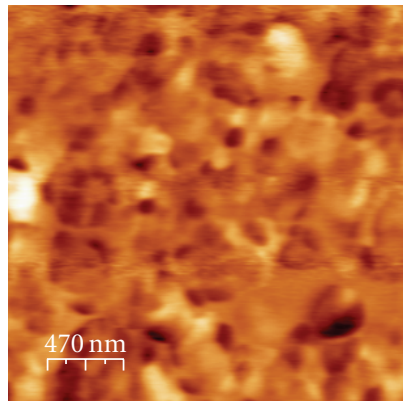

(b)

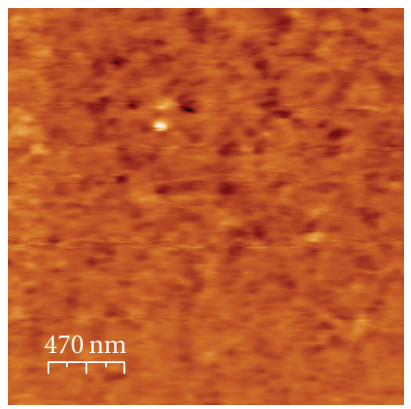

(c)

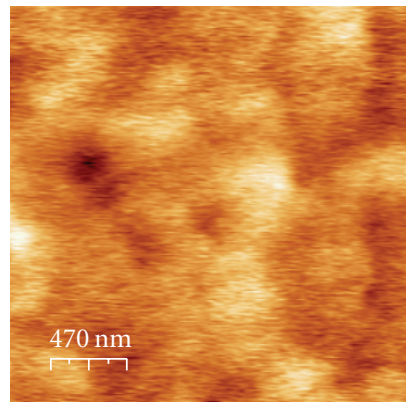

(d)

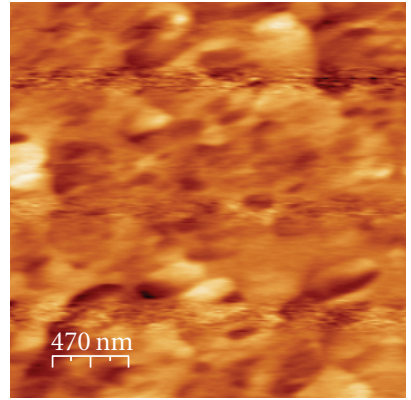

(e)

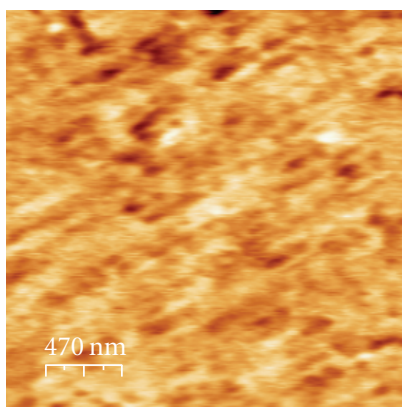

(f)

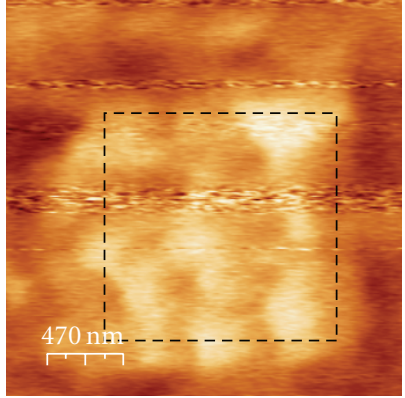

(g)

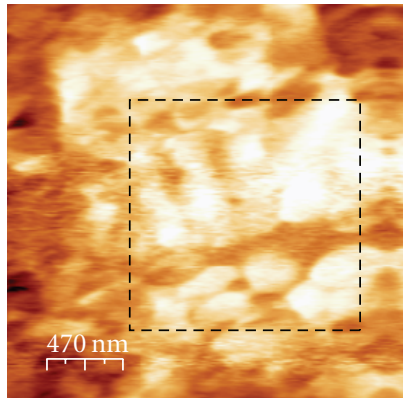

(h)

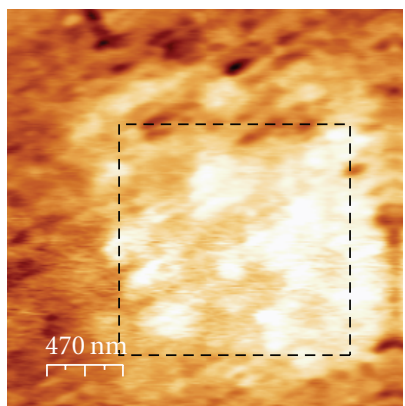

(i)

FIgure 3: Piezoresponse images of the PZT series films. (a) (c) are piezoresponse images for PZT, PZT-BT1, and PZT-BT5 as-deposited films, respectively; (d) (f) show piezoresponse images on fatigued PZT series films; (g) (i) show corresponding poling effects on these films. The black frame represents poling area produced by moving the cantilever while applying a positive $20 \mathrm{~V}_{\mathrm{dc}}$ voltage. Unswitchable domains appear as dark color.

height $\phi$. As a result, the electrons ejected from tip may screen applied voltage and greatly decrease the $V_{\mathrm{dc}}$ of the PZT film as a reverse bias voltage, resulting in a strong imprint in piezoloop of PZT film. The mechanism for electron penetration may come from the high field induced by unscreened embryo domains, which is probably expressed by FowlerNordhein tunneling as [22]

$$
J=C_{\mathrm{FN}} E_{\mathrm{bc}}^{2} \exp \left[-\frac{4 \sqrt{2 m^{*}}\left(q \phi_{b}\right)^{3 / 2}}{3 q \xi E_{\mathrm{bc}}}\right]
$$

where $C_{\mathrm{FN}}$ is the Fowler-Nordheim coefficient which depends on the barrier height $\phi_{B}$ and the the electron effective mass $m^{*}$ at the interface. Under such a high tunneling current, the penetration of the charge can be expected especially near the nucleation sites.
Comparing the piezoloops shown in Figures 3(a), 3(b), and $3(\mathrm{c})$, an apparent difference in loop shapes after fatigue was observed. After $3 \times 10^{8}$ fatigue cycles, the pinched degree for seeded films was much more alleviated as compared with unseeded PZT film. The slopes of the piezoloop near the coercive field for seeded films decreased moderately, which indicates that the domains in the area just under the tip were not largely frozen. It implied that there were different fatigue processes between seeded and unseeded films.

Some authors have suggested oxygen vacancy migration or accumulation near the film electrode will result in electrical fatigue $[3,5,6]$. To verify the role of oxygen vacancies in fatigue course, the PZT sample was annealed in nitrogen atmosphere for half an hour. The oxygen vacancies could not be compensated by ambient oxygen during cooling process, resulting in the distortion of the oxygen octahedron which would deteriorate the ferroelectric properties. As shown in 


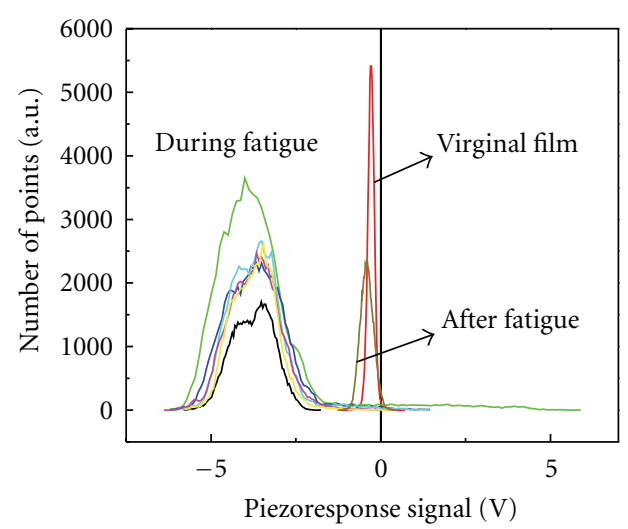

(a)

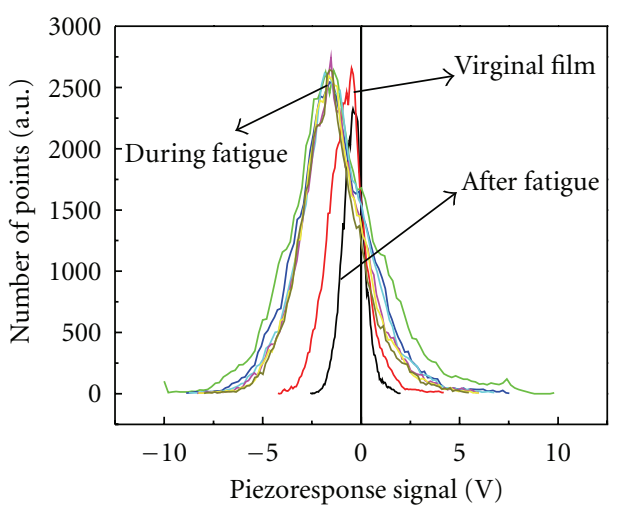

(b)

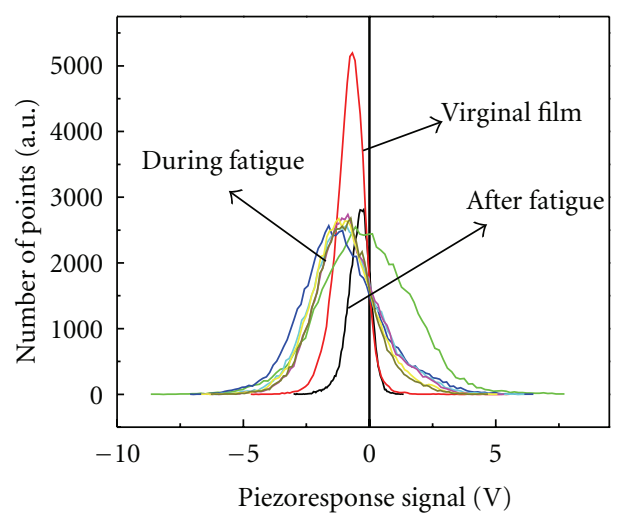

(c)

FIGURE 4: Piezoelectric histograms of three kinds of PZT films. Three types of histograms about virginal film, during fatigue and after fatigue, are shown, respectively, in (a) PZT, (b) PZT-BT1, and (c) PZT-BT5 films.

the inset of Figure 2(c), the remnant polarization of nitrogen-processed sample before fatigue decreased to one-third of the virginal sample. After $3 \times 10^{8}$ electrical cycles, the fatigued loop of this sample was severely pinched, but from the normalized viewpoint, it is unlikely that the oxygen vacancies play a key role in the fatigue mechanism.

3.2. Dynamic Scanning Fatigue Studies. To further investigate the fatigue effect in these PZT films, a further experiment was carried out. A $2.35 \times 2.35 \mu \mathrm{m}^{2}$ square area was scanned for 6 cycles (about 50 minutes) by a conductive tip on which high voltage was applied to induce repeated polarization switching. We briefly call this mode scanning fatigue, which parallels the standing fatigue as presented earlier in Figure 3. The scanning fatigue mode is equivalent to the application of $5 \times 10^{3}$ electrical pulses to each pixel on surface of the film. After the fatigue operation, a new cantilever was used to study the same original sample area. Then, a smaller area inside the original fatigued area was poled with high positive voltage to observe the domain pinning in the fatigued area. These results are shown in Figure 3. It is noticed that pure PZT film shows a considerable change in the piezoresponse pattern of the fatigued area, while PZT films with BT nanoparticles maintained their domain distributions. The right column of Figure 3 shows the piezoresponse images of these films, where a positive $20 V_{\mathrm{dc}}$ voltage was applied through the scanning tip to induce reorientations on the corresponding fatigued area. For pure PZT film, it is obvious that some of the domains could not be reoriented and still exhibit its original piezoelectric signals. This is a strong indication that there were domains pinning around these regions [23]. In the case of the PZT films, with nanoparticles, the area of unswitchable region was much smaller as compared to PZT film and further for PZT-BT5 film, the domain populations inside the black frame could even be switched freely.

The piezohistograms of these films in as-deposited, during-fatigue, and after-fatigue states obtained from corresponding PFM images are shown in Figure 4. It is complex to analyze the during-fatigue piezoresponse image due to an intricate domain switching case which is involved into it. Theoretically, when we consider the first harmonic piezoresponse during domain switching as a sine ac signal is used, the amplitude of piezoelectric displacement will present two same segments corresponding to one period of the ac signal. Thus, the whole first harmonic signal acquired from upper layer of the measured object should be zero. In fact, some signal strength must be present due to a highly inhomogeneous electric field beneath the tip. However, we preferably consider that this first harmonic signal results from the piezoelectric contribution at deeper region just beneath the upper one in the surface. 


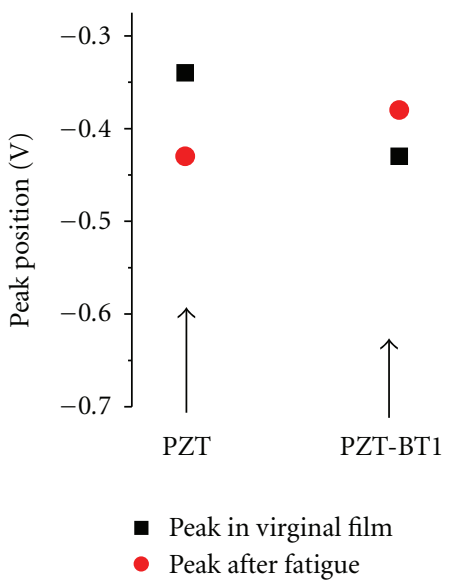

(a)
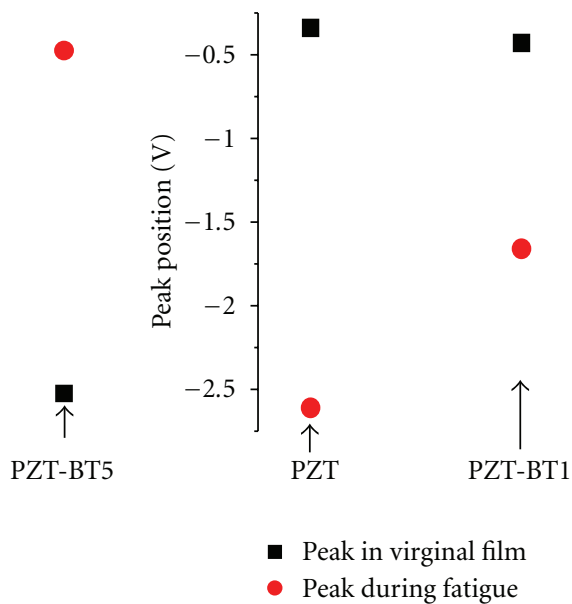

(b)

Figure 5: Peak offset in corresponding histogram between (a) virginal film and after-fatigue film, (b) virginal film and during-fatigue film.

Generally, piezohistogram describes statistical distribution of domain population and provide a tangible reference for domain orientation. The peak of a piezohistogfam refers to the most probable domain configuration, and its width indicates possible number of domain variations. The height of the curve is highly width-dependent, because the number of events is closely related to span of the interval. So, we ignore the variation of height in these histograms. Analysis of piezohistograms for virgin films reveals that these PZT films exhibit self-polarization effect. It is due to the strong built-in field near the bottom electrode formed by the trapped electrons and ionic defects near the film-bottom electrode interface [24]. This effect produced an additional internal electric field with a determinate direction, inducing mainly downward domains in this area, as shown in Figure 4. Furthermore, a large offset of the piezohistogram is observed for pure PZT film when a large ac voltage is applied to excite domain switching. In contrast to the pure PZT film, the PZT films with nanoparticles have smaller offset even almost zero for PZT-BT5 film in that moment as shown in Figure 4(c). After application of electric cycles, the pure PZT film shows a negative offset, which indicates that its self-polarization will be strengthened. On the contrary, films with nanoparticles present small positive offsets as shown in Figures 4(b) and $4(c)$. Then, alleviation in their self-polarization can be expected.

Concerning sol-gel-derived PZT films, the presence of built-in bias field near bottom electrode can result in selfpolarization. It is both probable that the injected electrons come from bottom electrode or from tip, and these two types of electrons induce reverse effect for self-polarization [24]. The results in Figure 5 follow that the aggravation of the self-polarization during fatigue probably results from the injection of electrons from the bottom electrode. It should be noted that the aggravation degree is different for these films. After the fatigue process ended, opposite offset shown in Figure 5(a) implies that the injected electrons are trapped in different region. For pure PZT film, they are located near the bottom electrode, and for films with nanoparticles, they stay near the surface.

To investigate the effect of injected electrons on switching character, piezoloops for PZT and PZT-BT5 films was carried out before and after scanning fatigue as shown in Figure 6 . Comparison of these two kinds of films reveals the scanning fatigue cause opposite shifts in the vertical direction. It also implies that charges are in different interface, resulting in opposite bias field. We find that this result is in good agreement with [23] measured on fatigue effect in PZT film [23].

We suggest that film microstructure may be responsible for the fatigue performance. In our previous work [13], detailed crystallization processes for these PZT films were described. For PZT films with nanoparticles, nucleation occurs at the surface of the bottom electrode, at the seed surfaces, and at the top film surface, which brings about a higher degree of perovskite crystallinity but a less orientated texture, that is, granular structure. For pure PZT film, the nucleation takes place only at the surface of the bottom electrode. Then, columnar structure can be expected.

The change of film grain morphology from granular to columnar may have an effect on the fatigue performance in seeded and unseeded films. Generally, grain boundaries act as current pass, and as a result, injected electrons may vanish faster in films with lots of grain boundaries. Thus, the injected electrons will not pin the domain reorientations in films with the addition of nanoparticles. Therefore, good fatigue endurance is present in films with nanoparticles rather than pure PZT film. It is interesting to note that this suggestion is not in well consistent with the results in the reference [25], in which greater polarization fatigue for a granular PZT film was reported.

We further suggest that the inferior fatigue performance in unseeded film is closely related to early crystallization process. When the pure PZT film was pyrolyzed at $400^{\circ} \mathrm{C}$ for $5 \mathrm{~min}$, at the $\mathrm{Pt} / \mathrm{PZT}$ interface, $\mathrm{a} \mathrm{Pt}_{x} \mathrm{~Pb}$ interlayer 


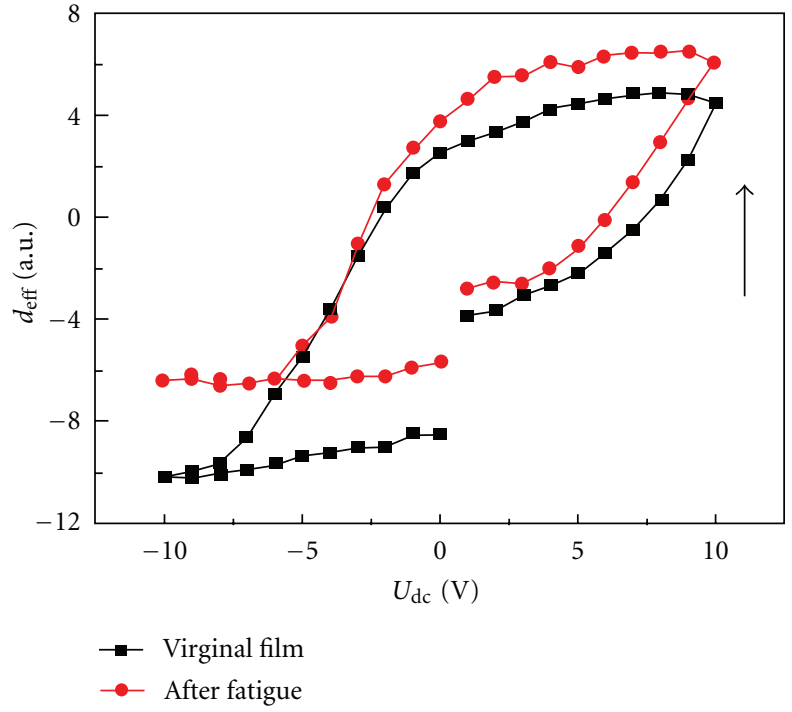

(a)

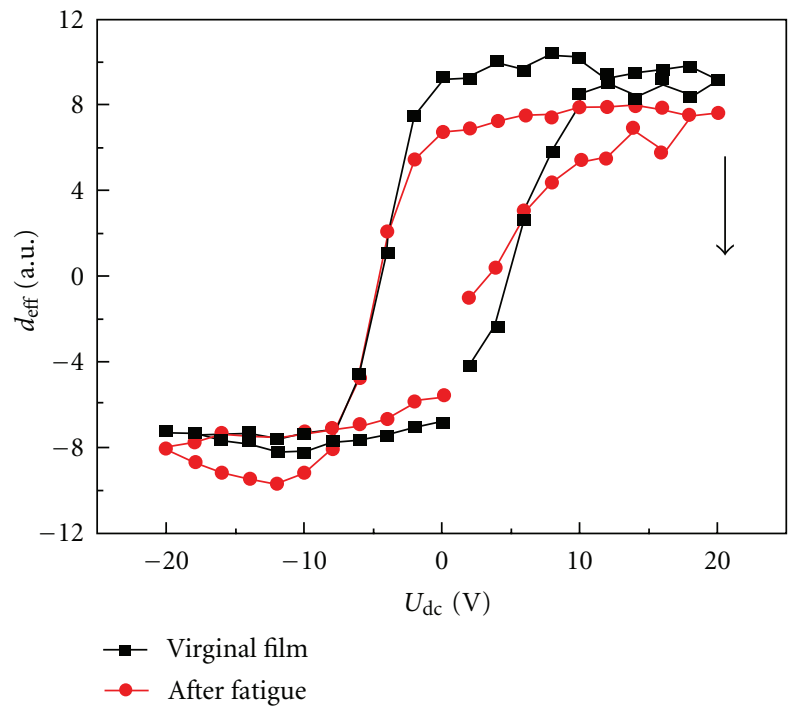

(b)

FIgURe 6: Variations of Piezoloop after fatigue for (a) unseeded and (b) seeded films.

was observed even though it disappeared in later period [13]. For seeded films pyrolyzed under the same conditions, the $\mathrm{Pt}_{x} \mathrm{~Pb}$ interlayer was reduced or not observed when seeds content increased to 5\%. Furthermore, there was evidence that the morphology of $\mathrm{Pt}_{x} \mathrm{~Pb}$ interlayer for PZT film was continuous while it was intermittent for PZT films with nanoparticles. Disappearance of the interlayer implies that the location with such a transition phase is prone to transform into heterogeneity which is easy to be penetrated by injected electrons. Besides this, electrons are more difficult to vanish in pure PZT film than in PZT films with nanoparticles so that the injected electrons are easy to pin the domain reorientation in pure PZT film. We believe that these are the reasons behind the inferior fatigue performance for pure PZT film. The possible incomplete compensation of depolarization field [7] did not to influence the electron injection, since we found in another experiment that static fatigue showed no frequency dependence.

\section{Conclusions}

In summary, we have demonstrated that polarization fatigue of PZT films depends on the addition of nanoseeds with piezoresponse force microscopy. Piezoresponse images and related piezoloops reveal that seeded films possess better fatigue endurance. We suggest that the film morphology and the microstructure of the bottom interface play key roles in the evolvement of the fatigue. Formation of the continuous $\mathrm{Pt}_{x} \mathrm{~Pb}$ interlayer in interface may damage the intactness of the bottom electrode although the interlayer disappears in latter crystallization. These areas probably act as the pipeline for penetration of the electrons, and the fatigue endurance degrades with the combinations between mobile carriers and these electrons. In addition, the fatigue performance may also be affected by film microstructure which will evolve from columnar to granular growth with the addition of nanoseeds.

\section{Acknowledgment}

The author would like to acknowledge sample preparation and some experimental results done at University of Avero.

\section{References}

[1] J. F. Scott and C. A. Paz De Araujo, "Ferroelectric memories," Science, vol. 246, no. 4936, pp. 1400-1405, 1989.

[2] G. H. Haertling, "Ferroelectric ceramics: history and technology," Journal of the American Ceramic Society, vol. 82, no. 4, pp. 797-818, 1999.

[3] M. Dawber and J. F. Scott, "A model for fatigue in ferroelectric perovskite thin films," Applied Physics Letters, vol. 76, no. 8, pp. 1060-1062, 2000.

[4] J. F. Scott, C. A. Araujo, B. M. Melnick, L. D. McMillan, and R. Zuleeg, "Quantitative measurement of space-charge effects in lead zirconate-titanate memories," Journal of Applied Physics, vol. 70, no. 1, pp. 382-388, 1991.

[5] J. F. Scott and M. Dawber, "Oxygen-vacancy ordering as a fatigue mechanism in perovskite ferroelectrics," Applied Physics Letters, vol. 76, no. 25, pp. 3801-3803, 2000.

[6] C. H. Park and D. J. Chadi, "Microscopic study of oxygenvacancy defects in ferroelectric perovskites," Physical Review $B$, vol. 57, no. 22, pp. R13961-R13964, 1998.

[7] D. C. Lupascu and U. Rabe, "Cyclic cluster growth in ferroelectric perovskites," Physical Review Letters, vol. 89, no. 18, pp. 187601/1-187601/4, 2002.

[8] A. K. Tagantsev, I. Stolichnov, E. L. Colla, and N. Setter, "Polarization fatigue in ferroelectric films: basic experimental findings, phenomenological scenarios, and microscopic features," Journal of Applied Physics, vol. 90, no. 3, pp. 1387-1402, 2001.

[9] W. L. Warren, D. Dimos, B. A. Tuttle, and D. M. Smyth, "Electronic and ionic trapping at domain walls in $\mathrm{BaTiO}_{3}$," Journal 
of the American Ceramic Society, vol. 77, no. 10, pp. 2753-2757, 1994.

[10] J. F. M. Cillessen, M. W. J. Prins, and R. M. Wolf, "Thickness dependence of the switching voltage in all-oxide ferroelectric thin-film capacitors prepared by pulsed laser deposition," Journal of Applied Physics, vol. 81, no. 6, pp. 2777-2783, 1997.

[11] X. Du and I. Chen, " Model experiments on fatigue of $\mathrm{Pb}$ $\left(\mathrm{Zr}_{0.53} \mathrm{Ti}_{0.47}\right) \mathrm{O}_{3}$ ferroelectric thin films," Applied Physics Letters , vol. 72, p. 1923, 1998.

[12] A. Wu, P. M. Vilarinho, I. M. Miranda Salvado, and J. L. Baptista, "Seeding studies in PZT thin films," Materials Research Bulletin, vol. 33, no. 1, pp. 59-68, 1998.

[13] A. Wu, P. M. Vilarinho, I. Reaney, and I. M. Miranda Salvado, "Early stages of crystallization of sol-gel-derived lead zirconate titanate thin films," Chemistry of Materials, vol. 15, no. 5, pp. 1147-1155, 2003.

[14] A. L. Kholkin, S. V. Kalinin, A. Roelofs, and A. Gruverman, "Review of ferroelectric domain imaging by Piezoresponse Force Microscopy," in Scanning Probe Microscopy: Electrical and Electromechanical Phenomena at the Nanoscale, S. Kalinin and A. Gruverman, Eds., Springer, New York, NY, USA, 2006.

[15] M. Alexe, C. Harnagea, D. Hesse, and U. Gösele, "Polarization imprint and size effects in mesoscopic ferroelectric structures," Applied Physics Letters, vol. 79, no. 2, pp. 242-244, 2001.

[16] S. Bühlmann, E. Colla, and P. Muralt, "Polarization reversal due to charge injection in ferroelectric films," Physical Review $B$, vol. 72, no. 21, Article ID 214120, 7 pages, 2005.

[17] G. Arlt, D. Hennings, and G. De With, "Dielectric properties of fine-grained barium titanate ceramics," Journal of Applied Physics, vol. 58, no. 4, pp. 1619-1625, 1985.

[18] E. L. Colla, I. Stolichnov, P. E. Bradely, and N. Setter, "Direct observation of inversely polarized frozen nanodomains in fatigued ferroelectric memory capacitors," Applied Physics Letters, vol. 82, no. 10, pp. 1604-1606, 2003.

[19] X. H. Du, U. Belegundu, and K. Uchino, "Crystal orientation dependence of piezoelectric properties in lead zirconate titanate: theoretical expectation for thin films," Japanese Journal of Applied Physics, Part 1, vol. 36, no. 9, pp. 5580-5587, 1997.

[20] S. V. Kalinin, A. Gruverman, and D. A. Bonnell, "Quantitative analysis of nanoscale switching in SrBi2Ta $2 \mathrm{O} 9$ thin films by piezoresponse force microscopy," Applied Physics Letters, vol. 85, no. 5, pp. 795-797, 2004.

[21] A. Wu, P. M. Vilarinho, V. V. Shvartsman, G. Suchaneck, and A. L. Kholkin, "Domain populations in lead zirconate titanate thin films of different compositions via piezoresponse force microscopy," Nanotechnology, vol. 16, no. 11, pp. 2587-2595, 2005.

[22] E. Bouyssou, P. Leduc, G. Guégan, and R. Jérisian, "Leakage current conduction in $\mathrm{IrO}_{2} / \mathrm{PZT} / \mathrm{Pt}$ structures," Journal of Physics, vol. 10, no. 1, pp. 317-320, 2005.

[23] X. J. Lou, M. Zhang, S. A. T. Redfern, and J. F. Scott, "Local phase decomposition as a cause of polarization fatigue in ferroelectric thin films," Physical Review Letters, vol. 97, no. 17, Article ID 177601, 2006.

[24] A. Gruverman, O. Auciello, and H. Tokumoto, "Nanoscale investigation of fatigue effects in $\mathrm{Pb}(\mathrm{Zr}, \mathrm{Ti}) \mathrm{O}_{3}$ films," Applied Physics Letters, vol. 69, no. 21, pp. 3191-3193, 1996.

[25] A. Gruverman, B. J. Rodriguez, R. J. Nemanich, and A. I. Kingon, "Nanoscale observation of photoinduced domain pinning and investigation of imprint behavior in ferroelectric thin films," Journal of Applied Physics, vol. 92, no. 5, p. 2734, 2002. 

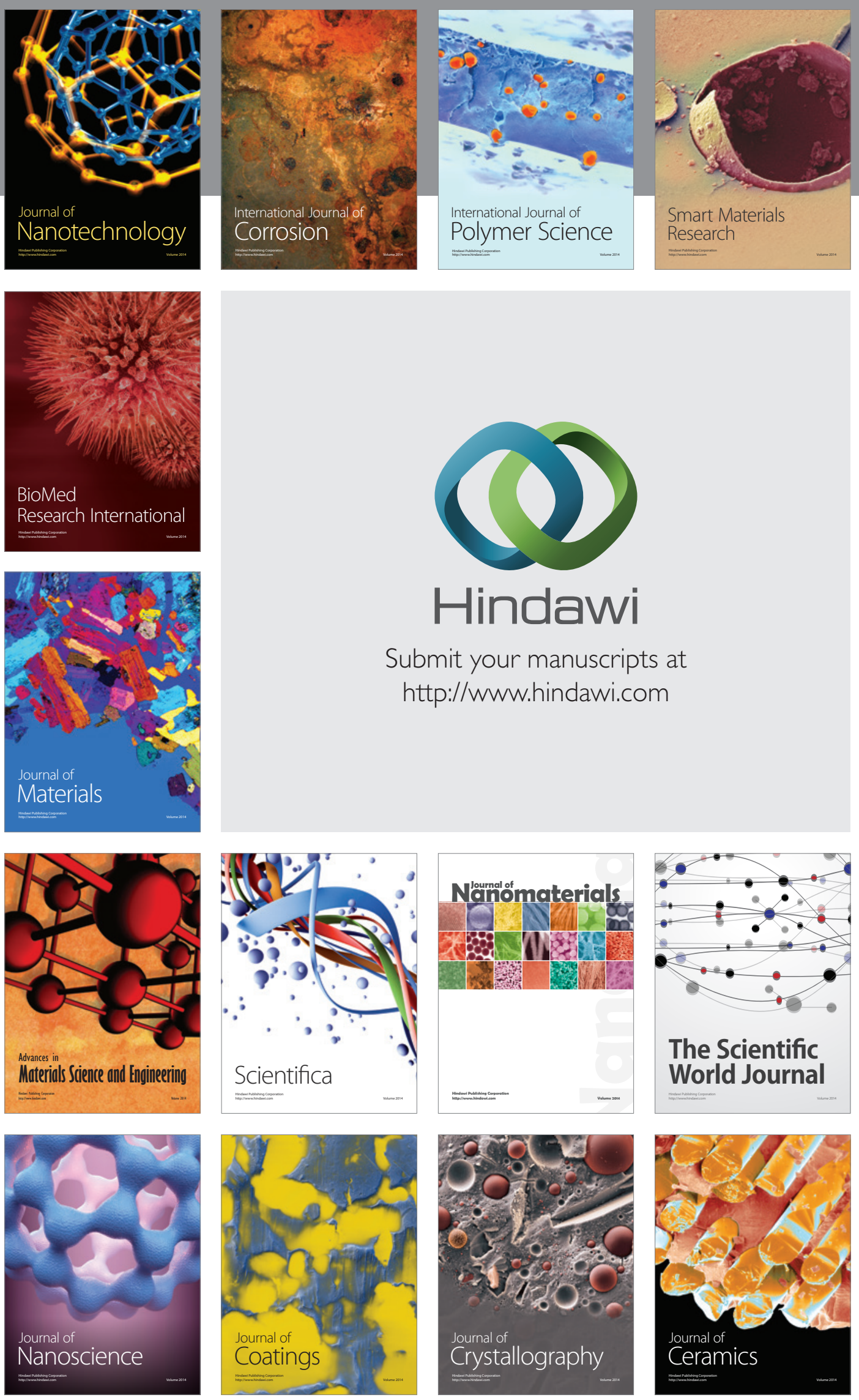

The Scientific World Journal

Submit your manuscripts at

http://www.hindawi.com

\section{World Journal}

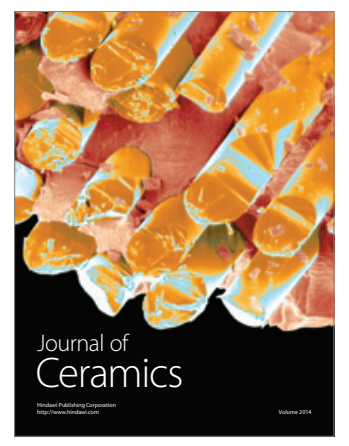

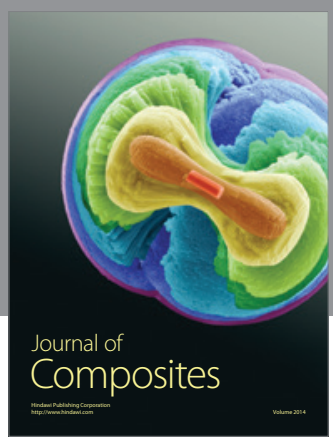
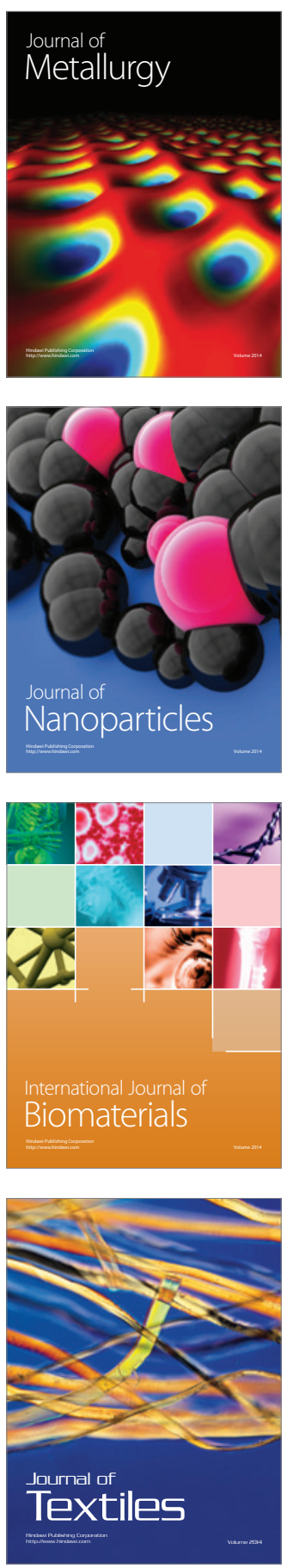